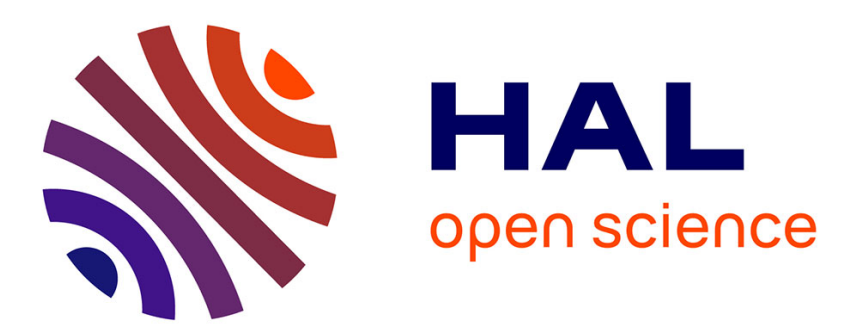

\title{
Segmenting junction regions without skeletonization using geodesic operators and the max-tree
}

\author{
Andrés Serna, Beatriz Marcotegui, Etienne Decencière
}

\section{To cite this version:}

Andrés Serna, Beatriz Marcotegui, Etienne Decencière. Segmenting junction regions without skeletonization using geodesic operators and the max-tree. 14th International Symposium on Mathematical Morphology and Its Applications to Signal and Image Processing, Jul 2019, Saarbrücken, Germany. 10.1007/978-3-030-20867-7_35. hal-02430543

\section{HAL Id: hal-02430543}

\section{https://hal-mines-paristech.archives-ouvertes.fr/hal-02430543}

Submitted on 7 Jan 2020

HAL is a multi-disciplinary open access archive for the deposit and dissemination of scientific research documents, whether they are published or not. The documents may come from teaching and research institutions in France or abroad, or from public or private research centers.
L'archive ouverte pluridisciplinaire HAL, est destinée au dépôt et à la diffusion de documents scientifiques de niveau recherche, publiés ou non, émanant des établissements d'enseignement et de recherche français ou étrangers, des laboratoires publics ou privés. 


\title{
Segmenting junction regions without skeletonization using geodesic operators and the max-tree
}

\author{
Andrés Serna $^{1}$, Beatriz Marcotegui ${ }^{1,2}$, and Etienne Decencière ${ }^{2}$ \\ 1 andres.serna@terra3d.fr \\ Terra3D - 36 Boulevard de la Bastille, 75012 Paris, France \\ http://www.terra3d.xyz/ \\ 2 \{beatriz.marcotegui, etienne.decenciere\}@mines-paristech.fr \\ MINES ParisTech, PSL Research University, \\ 35 rue Saint Honoré - Fontainebleau, France \\ http://cmm.mines-paristech.fr
}

\begin{abstract}
In a $2 \mathrm{D}$ skeleton, a junction region indicates a connected component (CC) where a path splits into two or more different branches. In several real applications, structures of interest such as vessels, cables, fibers, wrinkles, etc. may be wider than one pixel. Since the user may be interested in the junction regions but not in the skeleton itself (e.g. in order to segment an object into single branches), it is reasonable to think about finding junction regions directly on objects avoiding skeletonization. In this paper we propose a solution to find junction regions directly on objects, which are usually elongated structures, but not necessary thin skeletons, with possible protrusions and noise. Our method is based on geodesic operators and is intended to work on binary objects without holes. In particular, our method is based on the analysis of the wavefront evolution during geodesic propagation. Our method is generic and does not require skeletonization. It provides an intuitive and straightforward way to filter short branches and protuberances. Moreover, an elegant and efficient implementation using a max-tree representation is proposed.
\end{abstract}

Keywords: triple points, bifurcation pixel, junction region, skeleton, branches, geodesic distance, geodesic extremities, h-Maxima, max-tree.

\section{Introduction}

In a 2D skeleton, a triple point or bifurcation pixel is a point where the skeleton splits into three different branches. If it splits into four or more branches, the more generic term of junction point is used. Finding junction points in skeletons is a classic problem, usually solved by applying iterative hit-or-miss operators with some special structuring elements $[17,18]$.

Extracting the skeleton from a given object is a well studied problem in the literature $[9,16,21,8,7,3,2]$. However, as far as we know, there is no universally established best skeleton method. 
In several applications, the user may be interested in the junction regions but not in the skeleton itself (e.g. in order to segment an object into single branches). In such a case, the skeleton computation is not needed.

In this work, we propose a method to find junction regions of an object without holes avoiding skeletonization. Our method is based on geodesic operators and provides an intuitive and straightforward way to filter out spurious short branches and protuberances. The paper is organized as follows: Section 2 reminds some basic notions. Section 3 introduces our proposed method to find junction regions on binary objects without holes. An elegant and efficient implementation using a max-tree is also proposed. Section 4 presents some results and a comparison with respect to a classic method based on skeletonization. Finally, Section 5 presents the conclusions and perspectives of the work.

\section{Basic notions}

The goal of this Section is reminding some basic notions related to the Euler characteristic, geodesic operators and the max-tree structure. These concepts will be used in the proposed method explained later in Section 3.

\subsection{Euler characteristic}

Let $I_{b}$ be a digital binary image $I_{b}: D \rightarrow V_{b}$, with $D \subset Z^{2}$ the image domain with a given connectivity and $V_{b}=\{0,1\}$ the set of binary values. The Euler characteristic refers to the number of connected components (CC) of image $I_{b}$ minus the number of holes of these CCs [6]. In this paper, we call binary object each single $\mathrm{CC}$ in image $I_{b}$. Considering the Euler characteristic of a single $\mathrm{CC}$ allows us to classify binary objects into two categories: porous and nonporous. On the one hand, non-porous objects (without holes) have an Euler characteristic equal to one. On the other hand, porous objects (with holes) have an Euler characteristic lower than or equal to zero. This work focuses on nonporous objects. In this context, non-porous objects are also known as simply connected sets or objects without holes.

\subsection{Geodesic operators: distance, extremities and diameter}

The geodesic propagation consists in an iterative displacement of a wavefront within an object from some starting seeds until the whole object is reached. The shape of the wavefront is defined by a given connectivity. Let $X$ be a single binary object and let $p$ and $q$ be two points belonging to $X$. The minimum number of geodesic iterations required to reach $q$ starting from $p$ (or vice versa) is called the geodesic distance $d_{X}(p, q)$. In other words, the geodesic distance $d_{X}(p, q)$ is the minimum length of a path joining $p$ and $q$ and included in $X$ [10]

Fig. 1 presents an example of geodesic distance from given pixels inside a synthetic binary object using 8-connectivity. The propagation starts from the two pixels marked with a green square in the center of Fig. 1(a). In the first 
iteration, the geodesic distance for the starting points is set to zero. In the second iteration, the distance for the first neighboring points within the object is set to one. In the third iteration, the distance to the second neighbors is set to two, and so on. The process ends when the whole object has been processed. In this example, the process finishes at the $75^{\text {th }}$ iteration at the end of the spiral (See Fig. 1(b)). The geodesic distance is only computed for the points belonging to the object, thus pixels outside the object are set to infinite (or an invalid distance value, -1 in this example). Note that the geodesic propagation can be implemented as iterative geodesic dilations. However, a more efficient implementation is possible using priority queues [22].

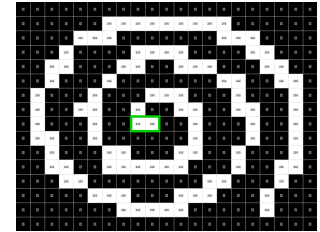

(a) Binary object

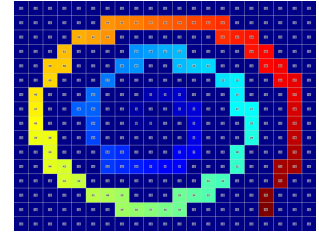

(b) Geodesic distance

Fig. 1. Geodesic distance from the highlighted pixels in the spiral.

We can compute $d_{X}(p, q)$ for all $p, q \in X$. Any couple of points where $d_{X}(p, q)$ reaches its global maximum are the geodesic extremities of $X$. To find the geodesic extremities of $X$, we use an efficient approximation based on the barycentric diameter proposed in $[11,12]$, which can be summarized as follows:

1. Let binary object $X$ be a single CC of image $I_{b}$ and its barycenter $B$.

2. Let $x$ be the farthest point of $X$ from $B$. Since $B$ does not necessary belong to $X$, the Euclidean distance is used to look for the farthest point $x$.

3. Compute the geodesic distance from $x$ inside $X$. The maximum value of this geodesic distance is $l^{1}(X)$ and the point where it is reached is $y_{1}$.

4. Compute the geodesic distance from $y_{1}$ inside $X$. The maximum of this new geodesic distance is $l^{2}(X)$ and the point where it is reached is $y_{2}$.

Based on this processing, $y_{1}$ and $y_{2}$ are estimations of the geodesic extremities of $X$ while $l^{2}(X)$ corresponds to its barycentric diameter. The authors [12] have shown that the barycentric diameter is a very good approximation of the geodesic diameter with an average error lower than $0.25 \%$ in a database of 51400 binary shapes. Fig. 2 illustrates the computation of the barycentric diameter in order to estimate two geodesic extremities and the geodesic diameter of an object.

\section{$2.3 \quad$ Max-tree}

Let $I$ be a digital gray-scale image $I: D \rightarrow V$, with $D \subset Z^{2}$ the image domain and $V=\{0, \ldots, R\}$ the set of gray-scale levels. A decomposition of $I$ can be obtained 


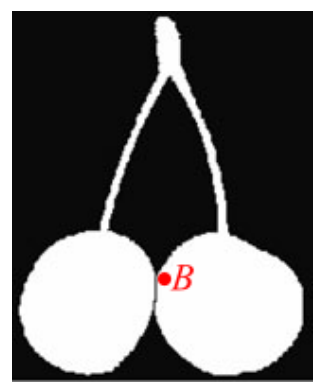

(a) Barycenter $B$

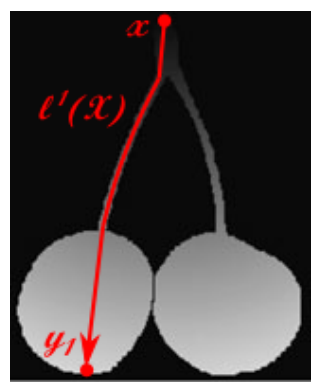

(b) $1^{\text {st }}$ propagation

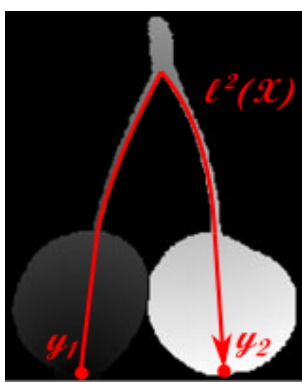

(c) $2^{\text {nd }}$ propagation

Fig. 2. Computation of the barycenter diameter in order to find two geodesic extremities of object $X$.

considering successive thresholds $T_{t}(I)=\{p \in D \mid I(p) \geq t\}$ for $t=\{0, \ldots, R\}$. Since this decomposition satisfies the inclusion property $T_{t}(I) \subseteq T_{t-1}(I), \forall t \in$ $[1, \ldots, R]$, it is possible to build a tree with the CCs of the sets $T_{t}(I)$. The max-tree (resp. min-tree) is a data structure in which each leaf corresponds to a maximum (resp. minimum) of the image and each node corresponds to a $\mathrm{CC}$ of a threshold of the image. This structure is particularly useful in the domain of connected operators [15]. The construction of the tree is based on the inclusion rule [5,14]. Several max-tree implementations are proposed in the literature $[5,14,23,13]$. In this paper, we use an improved version of the fast max-tree implementation proposed in [4].

Let us explain the max-tree construction with the toy example of Fig. 3. For this example 4-connectivity is used. Consider the image 3(a) containing 8 flatzones enumerated from $a$ to $h$ (in lower-case letters). Gray regions correspond to zero valued pixels. Figures 3(d) to 3(i) present the successive thresholds from $T_{1}(I)$ to $T_{6}(I)$, respectively. Fig. 3(c) presents the corresponding max-tree built from those successive thresholds. Note that a given threshold may be composed by several CCs, enumerated from $A$ to $H$ (in upper-case letters). For example, threshold $T_{4}(I)$ has two CCs $D$ and $G$. Moreover, a CC may be related to several flat-zones. For example, node $D$ in the max-tree is associated to flatzones $d, e$ and $f$ in the input image. The max-tree construction starts at the lowest level $t=0$, corresponding to the root of the tree. The first level is composed by threshold $T_{1}(I)$ containing CC $A$ in Fig. 3(d). Threshold $T_{2}(I)$ is composed by single CC $B$ while $T_{3}(I)$ is composed by $C$. Following the inclusion rule, $T_{3}(I) \subseteq T_{2}(I) \subseteq T_{1}(I)$, we add $B$ and $C$ to the main trunk of the tree. Node $C$ contains two children since threshold $T_{4}(I)$ in Fig. 3(g) contains two CCs $D$ and $G$. The construction of these two branches is straightforward since $F \subseteq E \subseteq D$ and $H \subseteq G$.

In addition, it is possible to associate a flat-zone in $I$ to a given node in the tree. Note that a node might be associated to several flat-zones. In fact, a given 


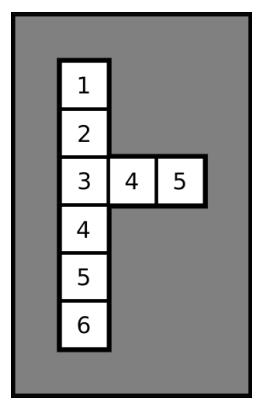

(a) Gray levels

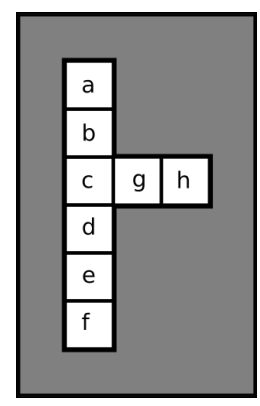

(b) Flat-zones

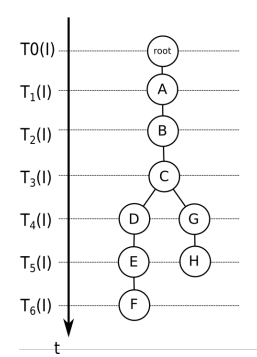

(c) Max-tree

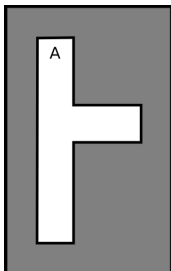

(d) $T_{1}(I)$

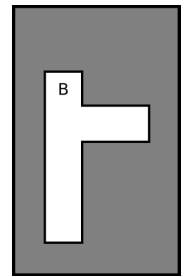

(e) $T_{2}(I)$

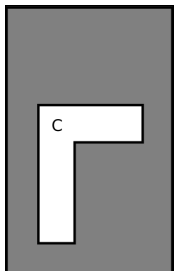

(f) $T_{3}(I)$

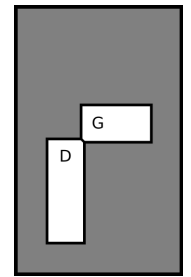

(g) $T_{4}(I)$

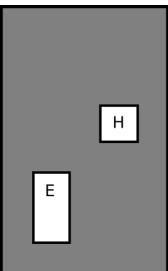

(h) $T_{5}(I)$

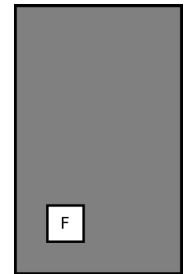

(i) $T_{6}(I)$

Fig. 3. Max-tree construction. The image is composed by 8 flat-zones enumerated from $a$ to $h$. Each node corresponds to a CC, enumerated from $A$ to $H$, of threshold $T_{t}(I)$. The max-tree contains two leafs corresponding to the two maxima at $f$ and $h$.

flat-zone at level $t$ is contained in the subset of $T_{t}(I)$ where gray level is equal to $t$ in the input image. For example, flat-zone $c$ in Fig. 3(b) corresponds to the pixels of $C$ in Fig. 3(f) where the gray level is equal to 3 in the input image : $c=\{p \in C \mid I(p)=3\}$.

In general, several filters can be applied to the tree (i.e. pruning leaves) and the output image is the restitution from the filtered tree. There exist several filtering rules to recompose the output image from the filtered max-tree $[1,14,20,19]$. The choice of the filtering rule depends on the application. In this paper, we will focus on the direct rule: all CCs $T_{t}(I)$ holding a given criterion are stacked to recompose the output image.

Let us illustrate the filtering process based on max-tree with the example of Fig. 4. In this case, we want to filter branches with contrast lower than or equal to 2 (this is equivalent to a h-Maxima filter with $h=2$ ). The contrast of a branch is defined as the gray-level difference between the first and the last node of the branch. This leads to remove the nodes $G$ and $H$ in Fig. 4(a). Fig. 4(b) shows the output image recomposed from the filtered max-tree using the direct rule. Fig. 4(c) shows the corresponding flat-zones filtered in the process.

Fig. 5 presents another example of image filtering using the max-tree structure. In this case, we want to remove nodes with less than 2 children. This leads to remove all the nodes except $C$ and the root of the tree (see Fig. 5(a)). Fig. 5(b) 


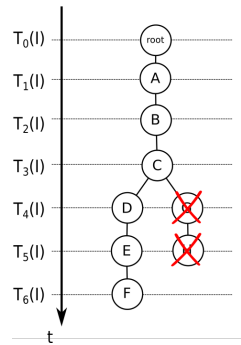

(a) Filtered maxtree

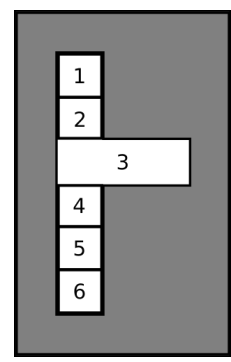

(b) Filtered image

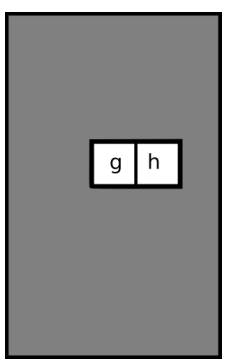

(c) FZ of nodes $G$ and $H$

Fig. 4. Max-tree based filtering on image of Fig. 3(a). Branches with contrast lower than or equal to 2 are removed. The output image is recomposed from the filtered max-tree using the direct rule. This is equivalent to a h-Maxima filter with $h=2$.

shows the output image recomposed from the filtered max-tree using the direct rule. Fig. $5(\mathrm{c})$ shows the flat-zone $c$ corresponding to remaining node $C$.

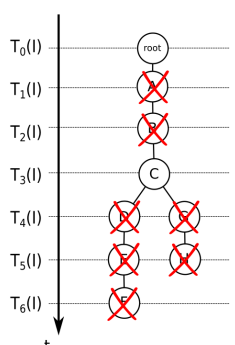

(a) Filtered maxtree

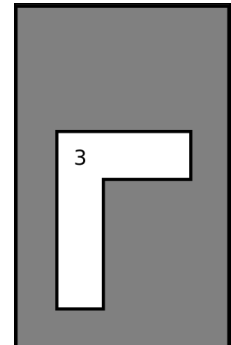

(b) Filtered image

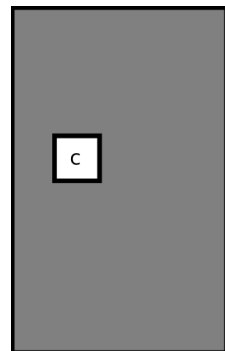

(c) Flat-zone of node $C$

Fig. 5. Max-tree based filtering on image of Fig. 3(a). Nodes with less than 2 children are removed. Output image is recomposed from the filtered max-tree. Fig. 5(c) shows the flat-zone $c$ corresponding to node $C$ in the input image.

\section{$3 \quad$ Finding junction regions}

A classic method to find junction regions can be summarized in Fig. 6 as follows:

1. Compute the skeleton of the input image (Fig. 6(a)). A great number of methods are proposed in the literature for this step $[9,16,21,8,7,3,2]$. 
2. Ideally, each junction region in the skeleton representation corresponds to an interesting intersection in the input object. In practice, there are often spurious junction regions associated to unwanted skeletal branches. If needed, these branches can be removed using a parametric pruning in order to get a clean skeleton (Fig. 6(b)).

3. Individual skeletal branches are obtained by suppressing junction regions from the skeleton (magenta points in Fig. 6(b)).

4. Junction regions are computed using the skeleton by influence zones (SKIZ) of the remaining skeleton (Fig. 6(c)) within the input object.

5. Finally, the SKIZ allows the segmentation in individual branches (Fig. 6(d)).

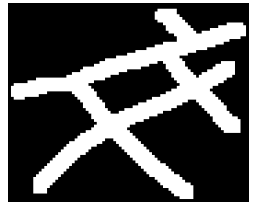

(a) Input object

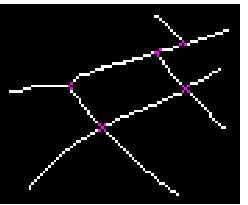

(b) Junctions on skeleton

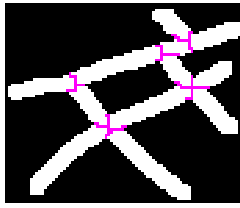

object

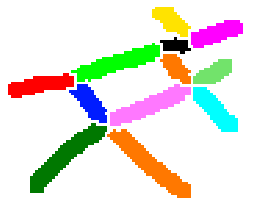

Segmented branches

Fig. 6. Extraction of junction regions using a method based on skeletonization.

In general, this classic method works well for thin and clean structures, but may fail on thick objects with bent branches and protrusions. A critical step is the choice of both the appropriate skeletonization algorithm and the pruning strategy in order to keep interesting branches. In several applications, the user is not particularly interested in the skeleton but in the junction regions. Thus, we propose to find junction regions directly on binary objects avoiding skeletonization. Our method is based on geodesic operators since the wavefronts of the geodesic propagation provide interesting information about junction regions. It is intended to work not only on thin objects but also on elongated structures with possible thick branches, protrusions and noise. Moreover, it provides an intuitive and straightforward way to filter out short branches and protuberances.

Consider a binary object without holes $X$, a geodesic propagation starting from a geodesic extremity $y$ and a max-tree $T$ built from the geodesic distance function $d_{X}(y)$. Let analyze the object of Fig. 3. In such a case, the propagation starts from the upper pixel of the object. When the propagation reaches a junction region, the wavefront splits from a single flat-zone $c$ at level $t=3$ to two separated flat-zones $d$ and $g$ at level $t=4$. This is represented by a node with two children in the max-tree. It is noteworthy that the flat-zone $c$, corresponding to node $C$, is a junction region. Informally, the number of branches in a junction region is equivalent to the number of children at its corresponding node in the max-tree representation. Since nodes with more than one child correspond to 
junction regions, we propose the following methodology to find junction regions on a binary object without holes $X$ :

1. Find a geodesic extremity $y$ of object $X$,

2. Compute the geodesic distance from $y$ inside $X$, called $d_{X}(y)$,

3. Construct a max-tree $T$ from $d_{X}(y)$,

4. (Optional) Compute a filtered tree $T^{\prime}$ by removing branches shorter than $h$,

5. Compute a filtered tree $T^{\prime \prime}$ by removing nodes with less than two children,

6. Junction regions $J$ are obtained as the flat-zones of $I$ corresponding to remaining nodes in filtered tree $T^{\prime \prime}$,

7. Individual branches $B$ are obtained by removing junction regions $J$ from the object $X: B=X \backslash J$.

Note that step 4 is optional and equivalent to applying a $h$-Maxima filter to the geodesic distance function. For the sake of efficiency, this filter can be computed directly on the max-tree by computing the contrast of each branch.

Fig. 7 illustrates our proposed method on a synthetic binary object. In this example 8-connectivity is used. The propagation starts from the lower left extremity of the object (green square in Fig. 7(a)). At the beginning, between iteration 1 and 12 in Fig. 7(b), each wavefront is composed of a single flat-zone. When the propagation reaches the first bifurcation at iteration 13 , the wavefront splits into two separate flat-zones. This bifurcation indicates the presence of a junction region. If we continue to the right of the image, we can see another junction region at iteration 16 . In this case, the wavefront splits producing three different flat-zones at value $t=17$.

It is noteworthy that each maximum in the geodesic distance function corresponds to an extremity of the object. As explained in Subsection 2.3, the contrast of a branch corresponds to its length. The h-Maxima operator can be used to filter maxima less contrasted than a given threshold $h$. This is a straightforward way to remove short and spurious extremities. Figures $7(\mathrm{c})$ to $7(\mathrm{f})$ present four examples of finding junction regions after h-Maxima filtering. Green points indicate the initial point of the geodesic distance computation, blue points indicate the regional maxima, and magenta points indicate the detected junction regions. In Fig. 7(c), the geodesic distance function contains four maxima, corresponding to the four extremities of the object (the starting point is of course an extremity, but it is a minimum because it is the starting point of the propagation). The object contains two junction regions at levels $t=13$ and $t=16$, respectively. In Fig. 7 (d), branches shorter than 3 pixels (h-Maxima filter with $h=3$ ) are filtered out. Note that the second extremity is not a maximum anymore and has been merged with the junction region. Please note here the impact of the connectivity on the branch length estimation. Although the upper left and the middle spikes of the object seem to have the same length, they are not filtered for the same value of h. If we use 4-connectivity instead of 8-connectivity, both extremities would be filtered at the same value of h. In Fig. 7(e), branches shorter than 4 pixels (h-Maxima filter with $h=4$ ) are filtered out. Note that only two extremities are still regional maxima of the image and only one junction region is detected. 
Finally, Fig. 7(f) shows a h-Maxima filter with $h=8$. Note that only the longest branch is still a maximum of the geodesic distance function. In this case, no junction regions are extracted.

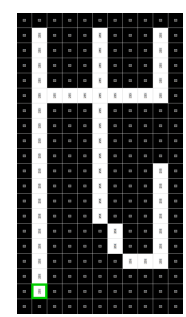

(a) Input

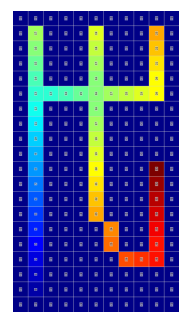

(b) $d_{X}(y)$

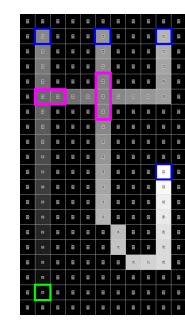

(c) $h=1$

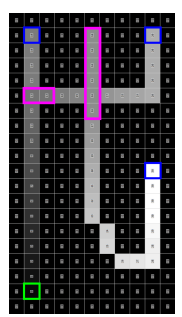

(d) $h=3$

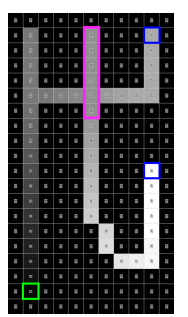

(e) $h=4$

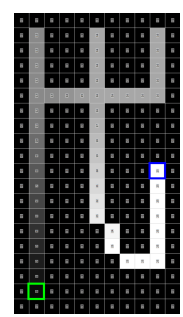

(f) $h=8$

Fig. 7. Junction regions on an object without holes. Green point indicates the initial seed of the geodesic distance, blue points indicate the regional maxima, and magenta points indicate the detected junction regions for several $h$ parameters.

\section{Results}

To evaluate our method, we present three tests on synthetic and real images. Our method is qualitatively compared to a classic method based on skeletonization (explained in Section 3). In order to make a fair comparison, the classical method is based on a "perfect" hand-made skeleton.

Fig. 8 illustrates an example of detection of junction regions on a binary object containing thin and thick branches. Fig. 8(c) presents the result using a classic method based on skeletonization. Fig. 8(d) presents the result of our proposed method. In both methods, branches shorter than 30 pixels have been filtered out. Note that our method is able to manage thick objects and the filtering strategy to remove non interesting branches is intrinsically taken into account in the max-tree representation. Note also that both methods are able to extract correctly the five junction regions, generating 10 individual branches. However, our method does not require any skeleton computation.

Fig. 9 illustrates a real top-view image of electric cables in a railway environment. Cables are interconnected at several locations. The goal is to segment the image into individual cable segments. Fig. 9(b) presents the segmentation result with our geodesic method considering all geodesic extremities $(h=1)$. Note that several junction regions are extracted due to short protrusions in the image. In fact, those protrusions correspond to cable insulators. In order to filter out these insulators, the segmentation is carried out considering only branches longer than 15 pixels, as shown in Fig. 9(c). 


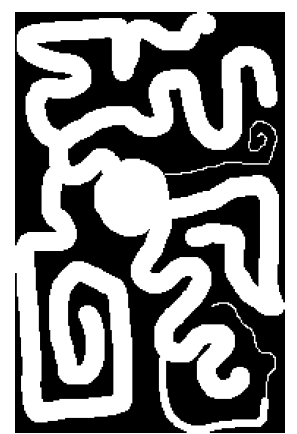

(a) Input

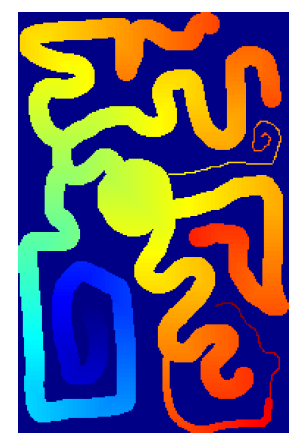

(b) Geodesic distance

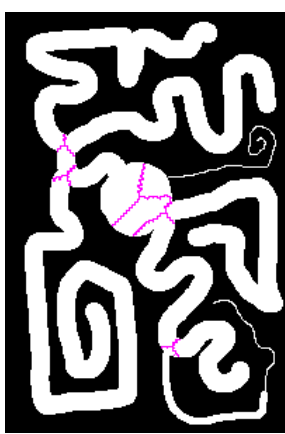

(c) Method based on skeletonization

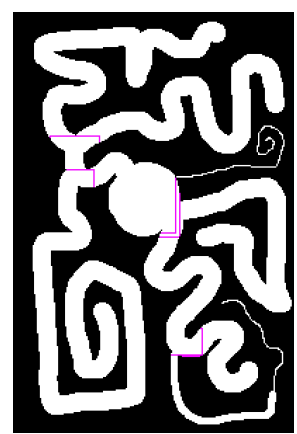

(d) Our geodesic method

Fig. 8. Junction regions using a classical method based on skeletonization and our proposed method. In both methods, branches shorter than 30 pixels are filtered out.

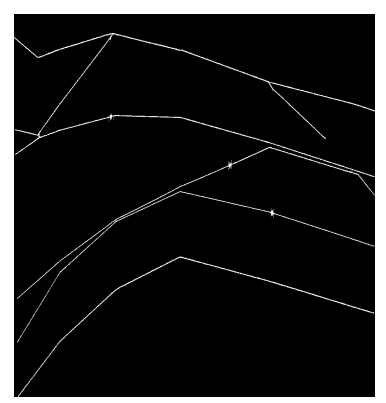

(a) Input image

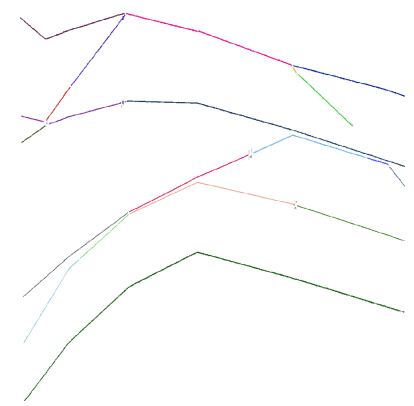

(b) $h=1$

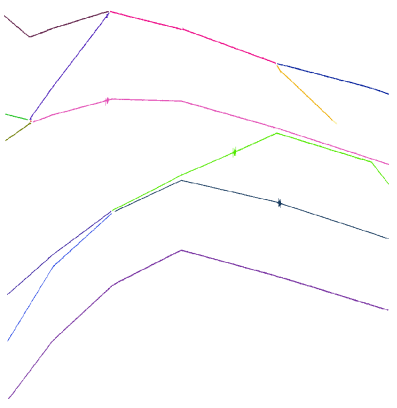

(c) $h=15$

Fig. 9. Segmentation of individual electric cables on a railway environment. Junction regions are extracted with our proposed geodesic method using two different $h$ parameters. Each color represents an individual cable.

Fig. 10 presents another example of electric cables in a railway environment. Fig. 10(a) presents the junction regions considering all branches $(h=1)$. Fig. 10(b) presents the junction regions considering only branches longer than 15 pixels $(h=15)$. In this case, cable insulators are not detected as junction regions, resulting in a correct segmentation of individual cables.

\section{Conclusions and perspectives}

We have proposed a method to find junction regions in objects without holes based on geodesic operators. In particular, our method is based on the analysis 


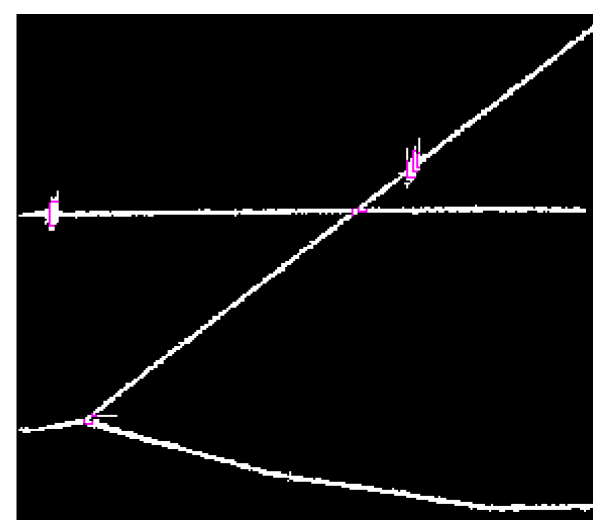

(a) $h=1$

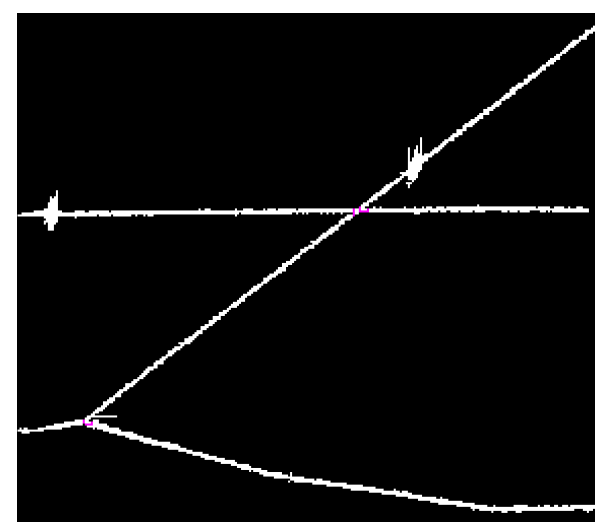

(b) $h=15$

Fig. 10. Segmentation of individual electric cables on a railway environment. Two different $h$ parameters have been used.

of the wavefront evolution in a geodesic propagation. Our method is generic and does not require skeletonization. It provides an intuitive and straightforward way to filter short extremities and protuberances. Moreover, an elegant and efficient implementation using a max-tree representation is proposed.

The problem with objects with holes is that the basic hypothesis: "the maxima on the geodesic distance correspond to the extremities of the object" does not hold anymore. In fact, a loop introduces the union of wavefronts coming from different branches, which introduces additional maxima on the geodesic distance function. We are currently working in a more generic method to extract junction regions on any binary object. The key step of such a method is replacing the max-tree structure by a more generic geodesic graph.

\section{References}

1. E. J. Breen and R. Jones. Attribute openings, thinnings, and granulometries. Computer Vision and Image Understanding, 64(3):377-389, 1996.

2. J. Chaussard. Topological tools for discrete shape analysis. $\mathrm{PhD}$ thesis, Université Paris-Est, dec 2010.

3. J. Chaussard, M. Couprie, and H. Talbot. Robust skeletonization using the discrete lambda-medial axis. Pattern Recognition Letters, 32(9):1384-1394, 72011.

4. J. Fabrizio and B. Marcotegui. Fast implementation of the ultimate opening. ISMM09, 9th International Symposium on Mathematical Morphology, pages 272281, 2009. Groningen, The Netherlands.

5. L. Garrido, A. Oliveras, and P. Salembier. Motion analysis of image sequences using connected operators. Proceedings of SPIE - The International Society for Optical Engineering, 3024:546-557, 011997. 
6. S. B. Gray. Local Properties of Binary Images in Two Dimensions. IEEE Transactions on Computers, C-20(5):551-561, may 1971.

7. W. H. Hesselink, M. Visser, and J. B. T. M. Roerdink. Euclidean Skeletons of 3D Data Sets in Linear Time by the Integer Medial Axis Transform. In Mathematical Morphology: 40 Years On, pages 259-268, Dordrecht, 2005. Springer Netherlands.

8. R. Kimmel, D. Shaked, N. Kiryati, and A. M. Bruckstein. Skeletonization via Distance Maps and Level Sets. Computer Vision and Image Understanding, 62(3):382391, 1995

9. C. Lantuéjoul. Skeletonization in quantitative metallography, volume 34, pages 107-135. Sijthoff and Noordhoff, 1980.

10. C. Lantuéjoul and S. Beucher. On the use of the geodesic metric in image analysis. Journal of Microscopy, 121(1):39-49, 1981.

11. V. Morard, E. Decencière, and P. Dokládal. Geodesic Attributes Thinnings and Thickenings Background : Attribute Thinnings. In ISMM'11, 10th international conference on Mathematical morphology, pages 200-211, 2011.

12. V. Morard, E. Decencière, and P. Dokládal. Efficient geodesic attribute thinnings based on the barycentric diameter. Journal of Mathematical Imaging and Vision, 46(1):128-142, 2013.

13. L. Najman and M. Couprie. Building the Component Tree in Quasi-Linear Time. IEEE Transactions on Image Processing, 15(11):3531-3539, 2006.

14. P. Salembier, A. Oliveras, and L. Garrido. Anti-extensive connected operators for image and sequence processing. IEEE Transactions on Image Processing, 7(4):555570, 041998.

15. P. Salembier and J. Serra. Flat zones filtering, connected operators, and filters by reconstruction. IEEE Transactions on Image Processing, 4:1153-1160, 1995.

16. J. Serra. Image analysis and mathematical morphology, volume 1. Academic Press, Orlando, FL, USA, 1982.

17. J. Serra. Image Analysis and Mathematical Morphology: Theoretical Advance, volume 2. Academic Press, 1988.

18. P. Soille. Morphological Image Analysis: Principles and Applications. SpringerVerlag, Secaucus, NJ, USA, 2003.

19. E. R. Urbach, J. B. T. M. Roerdink, and M. H. F. Wilkinson. Connected Shape-Size Pattern Spectra for Rotation and Scale-Invariant Classification of Gray-Scale Images. IEEE Transactions on Pattern Analysis and Machine Intelligence, 29(2):272285,2007

20. E. R. Urbach and M. H. F. Wilkinson. Shape-Only Granulometries and GrayScale Shape Filters. In ISMM'02, 8th International Symposium on Mathematical Morphology, pages 305-314, 2002.

21. L. Vincent. Efficient computation of various types of skeletons. In Medical Imaging V: Image Processing, volume 1445, pages 297-312. International Society for Optics and Photonics, 1991.

22. L. Vincent. Morphological grayscale reconstruction in image analysis: efficient algorithms and applications. IEEE Transactions on Image Processing, 2:176-201, 1993.

23. X. Huang and M. Fisher and D. J. Smith. An Efficient Implementation of Max Tree with Linked List and Hash Table. In DICTA, 2003. 